\title{
Pulse Function for Control of the Coherent Excitation in Stimulated Raman Spectroscopy
}

\author{
S. A. MALINOVSKAYA \\ MCTP, FOCUS Center, and Department of Physics, University of Michigan, \\ Ann Arbor, MI 48109, USA
}

Received 5 November 2003; accepted 30 August 2004

Published online 23 November 2004 in Wiley InterScience (www.interscience.wiley.com).

DOI 10.1002/qua.20393

\begin{abstract}
A selective excitation of closely spaced Raman transitions using femtosecond pulse shaping is studied within a semiclassical approach. An analytical pulse function is proposed having duration $T$ that selectively excites one of two transitions separated by a frequency interval less than $1 / T$. It is shown that, for intense fields, both the field amplitude and pulse frequency spectrum have to be carefully chosen to achieve the control of excitations. (c) 2004 Wiley Periodicals, Inc. Int J Quantum Chem 102: 313-317, 2005
\end{abstract}

Key words: mode selective excitation; femtosecond pulse control; coherent states; stimulated Raman scattering

\section{Introduction}

$\mathbf{U}$ nprecedented insight has been achieved into the nature of the dynamics of molecules, including biological polyatomic species, through the latest investigations using new-generation light sources. Wan et al. [1] used femtosecond lasers to directly observe the femtosecond dynamics of charge transport process occurring between bases within duplex DNA. By monitoring the population of an initially excited isomer of adenine, one can investigate the charge transfer process and measure the rate of the reaction. Ihee et al. [2] achieved imaging of complex chemical reactions including

This article was presented at the 11th International Congress of Quantum Chemistry held at Bonn from July 20 to July 26, 2003. the ring opening of a cyclic hydrocarbon with the implementation of ultrashort pulses of electrons. The ring opening reaction was studied by femtosecond spectroscopy in Refs. [3, 4]. Niikura et al. [5] elucidated a control mechanism for the selective molecular dissociation in homopolar molecules induced by a strong nonresonant laser field. Future perspectives have been discovered for studies of molecular structure and dynamics in light of advances in attosecond pulse technology [6-9].

A two-level quantum system can often serve to model laser-matter interactions. It is applicable to a variety of quantum problems, particularly to modeselective stimulated Raman spectroscopy, where ultrafast laser pulses are shaped to control vibrational excitations (see, e.g., Refs. [10-15]). It is noteworthy that the duration of the pulse is shorter than a typical vibrational period of a molecule, which cor- 
responds to an impulsive regime of interaction [16]. For weakly coupled vibrational modes and for broad-band excitation pulses, the problem of gain enhancement into a predetermined Raman band maps into the problem of enhancement of the $\mathrm{CO}^{-}$ herence of the two levels involved in the transition. This problem was addressed previously [17], but the proposed pulse intensity was not positive definite for all times. Here we present new solutions where the pulse intensity is constrained to be nonnegative. We address the shape and intensity a pulse should have to build up maximum coherence of one of two uncoupled two-level systems and zero coherence of another. The crucial condition in this problem is that the frequency difference of selectively excited transitions within two two-level systems is smaller than the pulse bandwidth, e.g., that $\Delta \omega \leq 1 / T$, where $T$ determines the duration of the pulse. This condition is relevant for a variety of molecules having close vibrational frequencies unresolved by transform-limited pulses.

In principle, it is possible to modulate the phase at different frequency components of the pulse, as it is done, e.g., in Refs. [18-20] for the population manipulation in CARS in perturbative regime. An antisymmetric function for the phase is used to invert the sign of the electric field at a given energy. The inversion induces constructive interference of the off-resonant components and maximizes the transition amplitude. In this work we retain the phase constant and propose a function for the intensity envelope. Design of the field amplitude of a broadband pulse may result in efficient control. The proposed function possesses free parameters carrying physical meaning. It is introduced in the frequency domain and designed to result in no excitation of one of the transitions in the weak-field regime. In strong fields numerical analysis reveals that to selectively excite one of two transitions with the designed pulse shape it is necessary to suitably choose the field amplitude.

\section{Basic Formalism}

To describe a molecule with two Raman-active vibrational modes we focus on a model of two two-level systems interacting with an intense offresonant femtosecond pulse. This pulse initiates stimulated Raman scattering via an off-resonant interaction with a virtual state. The FWHM of the pulse is significantly larger than the frequency difference of the two-level systems. This implies that the Stokes component of the field is supplied by the

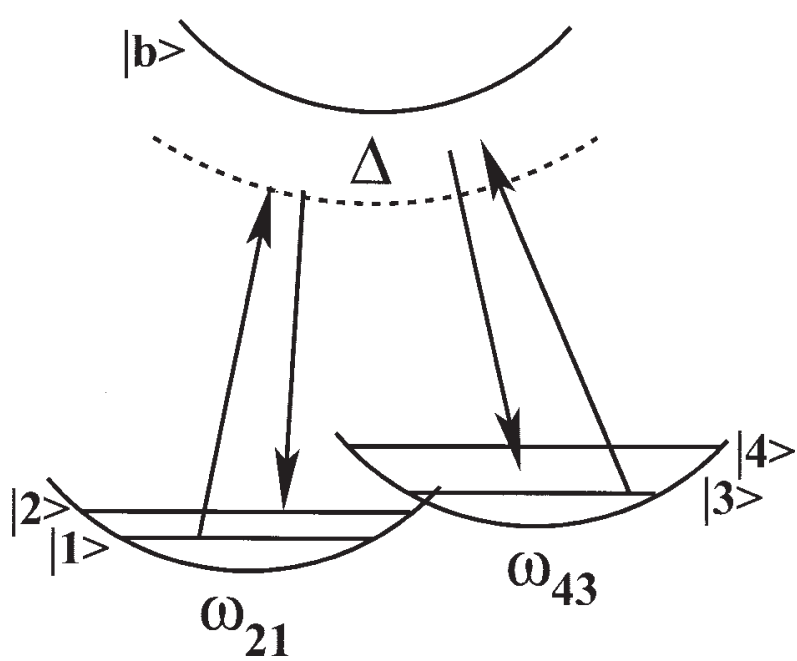

FIGURE 1. Schematic picture of a model system consisting of two two-level systems having frequencies $\omega_{21}$ and $\omega_{43}$. Initially, the lower levels are populated evenly. The uncoupled transitions are driven by an off-resonant femtosecond pulse.

same pulse. The goal of the present work is to determine a pulse shape that provides the control of excitations resulting in maximum coherence of one two-level system and zero coherence of another.

A semiclassical model of laser-molecule interactions is used. The model is represented schematically in Figure 1 where $\omega_{21}$ is the frequency of the 1-2 system with the coherence $\left|\rho_{12}\right|$ selected to be suppressed, and $\omega_{43}$ that of the 3-4 system with the coherence $\left|\rho_{34}\right|$ to be enhanced. Initially only lower levels $|1\rangle$ and $|3\rangle$ of both two-level systems are populated, and the populations of these levels are equal. The time evolution of two two-level systems is described in terms of probability amplitudes, which are written in the interaction representation as

$\dot{a}_{j}=i \frac{\Omega_{j}}{4 \Delta} \sum_{j^{\prime}=1}^{4} \Omega_{j^{\prime}}^{*} e^{-\left(\alpha_{j}-\alpha_{j^{\prime}}\right) \omega t} a_{j^{\prime}}, \quad \Omega_{j}=-\frac{\mu_{j b} E_{p 0}(t)}{\hbar}$.

Here $\alpha_{j} \omega$ is the frequency of a single level, such that, e.g., $\left(\alpha_{2}-\alpha_{1}\right) \omega=\omega_{21}, \Omega_{j}$ is a Rabi frequency, $\mu_{j b}$ is a dipole moment matrix element, $E_{p 0}(t)$ is the pulse envelope, and $\Delta$ is the detuning of the frequency of the pulse from the frequency of the virtual state $|b\rangle$. Note that the pulse envelope $E_{p 0}(t)$ is the same for all transitions. The Rabi frequencies may differ owing to different dipole moment matrix elements.

The system of coupled differential equations (1) is derived from the time-dependent Schrödinger equation with Hamiltonian [21]: 


$$
H=\frac{\hbar}{2}\left(\begin{array}{ccccc}
\alpha_{1} \omega & 0 & 0 & 0 & 2 \Omega_{1} \cos \left(\omega_{p} t\right) \\
0 & \alpha_{2} \omega & 0 & 0 & 2 \Omega_{2} \cos \left(\omega_{p} t\right) \\
0 & 0 & \alpha_{3} \omega & 0 & 2 \Omega_{3} \cos \left(\omega_{p} t\right) \\
0 & 0 & 0 & \alpha_{4} \omega & 2 \Omega_{4} \cos \left(\omega_{p} t\right) \\
2 \Omega_{1}^{*} \cos \left(\omega_{p} t\right) & 2 \Omega_{2}^{*} \cos \left(\omega_{p} t\right) & 2 \Omega_{3}^{*} \cos \left(\omega_{p} t\right) & 2 \Omega_{4}^{*} \cos \left(\omega_{p} t\right) & E_{b}
\end{array}\right)
$$

where $\omega_{p}$ is the laser field carrier frequency. By adiabatically eliminating state $|b\rangle$ within the rotating wave approximation, we arrive at Eqs. (1). In this work we discuss the case of uncoupled twolevel systems such that the probability for the population flow from one system to another via the external field is zero. Then Eqs. (1) are represented by two independent systems of coupled differential equations with two variables.

We propose an analytical function for the intensity envelope included in the dynamical equations (1) for the probability amplitudes. It is easiest to choose this function in the frequency domain. To enhance excitation at frequency $\omega_{43}$ and suppress excitation at frequency $\omega_{21}$, we choose

$$
\tilde{I}(\omega)=I_{0}\left(e^{-\left(T^{2}+T_{1}^{2}\right) \omega^{2}}+e^{-\left(\omega-\omega_{43}\right)^{2} T^{2}}\left(1-e^{-\left(\omega-\omega_{21}\right)^{2} T_{1}^{2}}\right)\right),
$$

where $T$ and $T_{1}$ are free parameters. When the modulation frequency of the pulse $\omega$ is equal to $\omega_{43}$ the intensity approaches maximum for a sufficiently large parameter $T_{1}$. For $\omega$ equal to $\omega_{21}$ the intensity is negligibly small.

The inverse Fourier transform of spectral density (3) is a complex function. To arrive at a physically acceptable temporal pulse function, we take the real part of the inverse Fourier transform, given by

$$
\begin{aligned}
& I(t)= I_{0} C\left(1+A / C \cos \left(\omega_{43} t\right)-B / C \cos \right. \\
&\left.\times\left(\left(\omega_{21}-\Delta \omega \frac{T^{2}}{\tau^{2}}\right) t\right)\right), A=(\sqrt{2} T)^{-1} e^{-\left(t^{2} / 4 T^{2}\right)}, \\
& B=(\sqrt{2} \tau)^{-1} e^{-\Delta \omega^{2} T^{2}\left(1-\left(T^{2} / \tau^{2}\right)\right)-\left(t^{2} / 4 \tau^{2}\right)}, C=(\sqrt{2} \tau)^{-1} e^{-\left(t^{2} / 4 \tau^{2}\right)}, \\
& \tau^{2}=T^{2}+T_{1}^{2}, \Delta \omega=\omega_{43}-\omega_{21} .
\end{aligned}
$$

The expression for field (4) is inserted in Eqs. (1) for the calculation of the probability amplitudes.

The intensity envelope as a function of time and frequency is drawn in Figures 2(a) and (b), respectively. The frequencies of the two-level systems are $\omega_{21}=1$ and $\omega_{43}=1.1$ in frequency units of $\omega_{21}$. The intensity of the field at $\omega_{43}$ and frequency region near $\omega_{21}$ over which $\tilde{I}(\omega)$ is small depend on $T_{1}$. The larger $T_{1}$, the greater is the selectivity for suppressing the $\omega_{21}$ frequency.

The solution of Eqs. (1) in the limit of a weak field using perturbation theory is

$$
\begin{array}{r}
a_{4}=i \frac{\mu_{4 b} \mu_{3 b}^{*}}{4 \Delta \hbar^{2}} \int_{-\infty}^{\infty} I(t) e^{i \omega_{43} t} d t=i \frac{\mu_{4 b} \mu_{3 b}^{*}}{4 \Delta \hbar^{2}} \tilde{I}\left(\omega_{43}\right), \\
a_{2}=i \frac{\mu_{2 b} \mu_{1 b}^{*}}{4 \Delta \hbar^{2}} \int_{-\infty}^{\infty} I(t) e^{i \omega_{21} t} d t=i \frac{\mu_{2 b} \mu_{1 b}^{*}}{4 \Delta \hbar^{2}} \tilde{I}\left(\omega_{21}\right) \\
\sim e^{-T^{2}\left(\omega_{43}+\omega_{21}\right)^{2}}+e^{-\Delta \omega^{2} T^{2}} e^{-\tau^{2}\left(2 \omega_{21}\right)^{2}} .
\end{array}
$$

The Fourier transform of the function $I(t)$ represented in Eq. (4) is not identical to Eq. (3), because we took the real part of the Fourier transform to arrive at (4). It now contains "counterrotating"
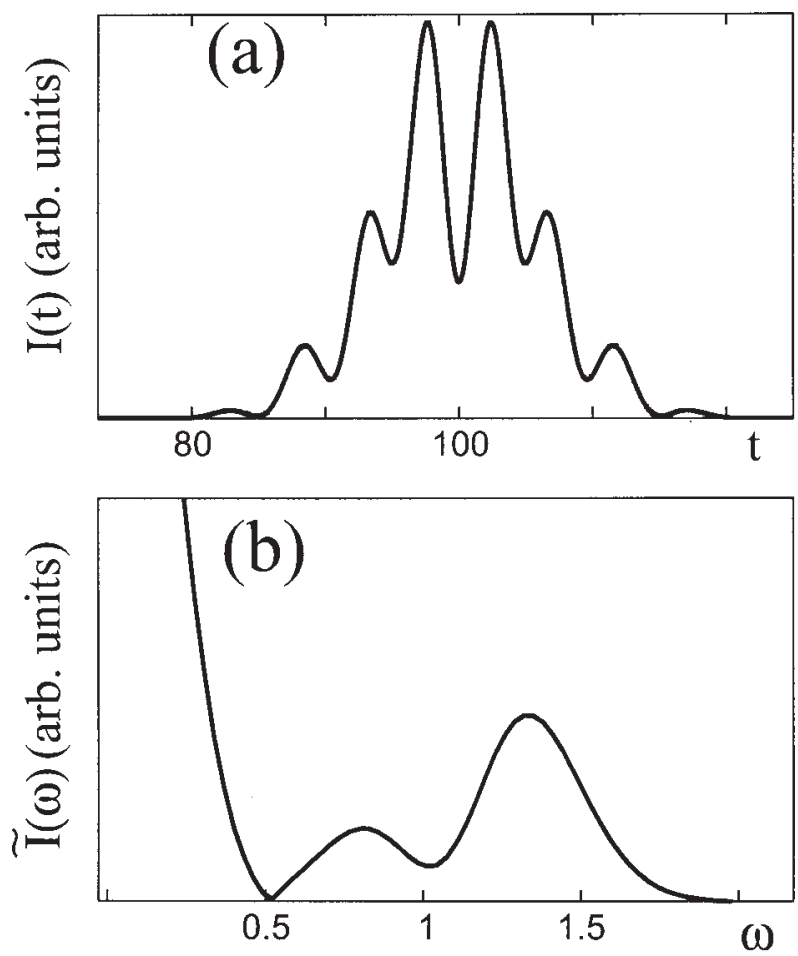

FIGURE 2. Intensity (a) temporal and (b) spectral profile for $T=3, T_{1}=3, \omega_{21}=1$, and $\omega_{43}=1.1$. 
terms, which are small for the chosen pulse shape. Thus, by construction [see also Fig. 2(b)], we have suppressed the 1-2 transition in the weak field limit. On the other hand, the excitation of the 3-4 transition is still weak owing to the perturbative nature of the solution.

\section{Numerical Results}

In this section we discuss the results of numerical calculations based on the exact solution of Eqs. (1). The numerical studies reveal the influence of the field parameters on the efficiency of the excitation of the two-level systems. Parameters for the system are taken from the experimental data on impulsive excitation of vibrational modes in the molecular gas $\mathrm{CO}_{2}$ [11]. In $\mathrm{CO}_{2}$ the frequencies of two selectively excited Raman modes are 36.8 and $42 \mathrm{THz}$. The full width at half-maximum (fwhm) of the applied intense pulse is taken equal to $18 \mathrm{THz}$. In our calculations the frequency $\omega_{21}$ is set equal to unity; in these units the frequency $\omega_{43}$ is equal to 1.1. From experimental data, we estimate that the parameter $T$ is about equal to 3 , in frequency units of $\omega_{21}^{-1}$. The intensity of the field is determined by the parameter $I_{0}$. The parameter $T_{1}$ is related to the width of the spectral dip in $\tilde{I}(\omega)$ centered at frequency $\omega_{21}$. Although a value of $T_{1} \gg T$ would provide the best selectivity, the choice for the parameter $T_{1}$ is strongly restricted by the requirement that the duration of the applied pulse be within a typical molecular vibrational period. It turns out that even for such values of $T_{1}$, it is possible to selectively excite one transition.

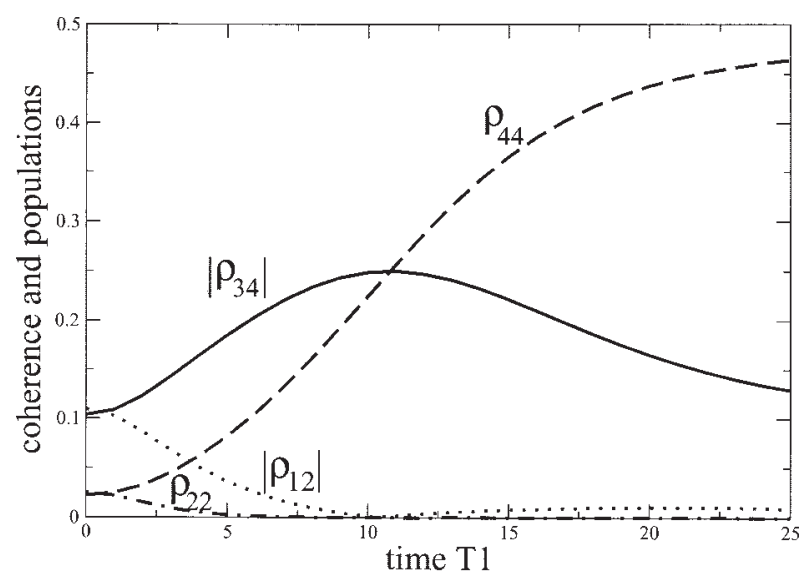

FIGURE 3. Coherence and populations of upper levels of the 3-4 and 1-2 two-level systems as a function of $T_{1}$ for $T=3, \omega_{21}=1, \omega_{43}=1.1$, and $I_{0}=\pi / 2$.

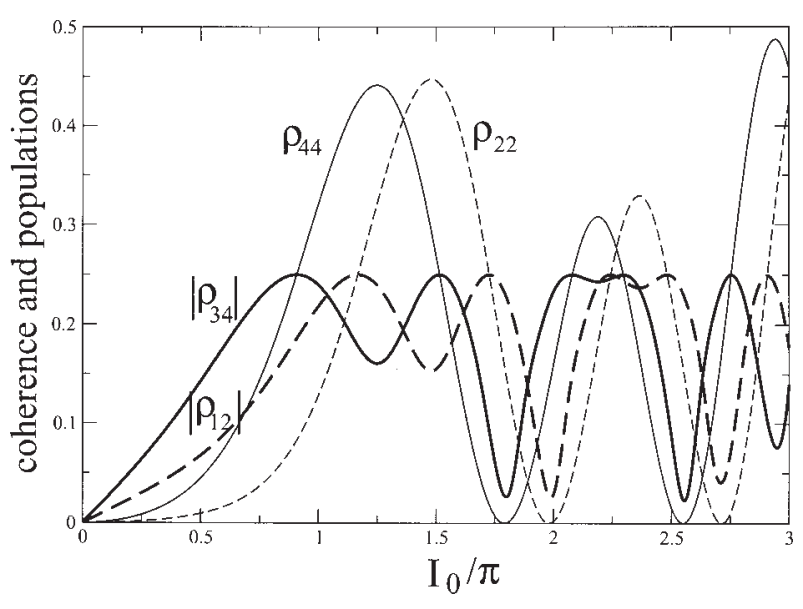

FIGURE 4. Intensity dependence of the coherences $\left|\rho_{12}\right|$ and $\left|\rho_{34}\right|$ and upper state populations $\rho_{22}$ and $\rho_{44}$. Maximum coherence of the 3-4 system and negligibly small coherence of the 1-2 system are observed for $I_{0}=2 \pi$ in the intensity region shown with $T=3$, $T_{1}=3$.

We calculated the population distribution and the coherence of the 1-2 and the 3-4 systems as a function of $T_{1}$ as shown in Figure 3. Bold solid and dotted lines depict the absolute value of the coherence $\left|\rho_{34}\right|$ of the excited 3-4 system and $\left|\rho_{12}\right|$ of the suppressed 1-2 system. Populations of the upper levels of both systems are shown by bold dashed and dot-dashed lines. The intensity of the field $I_{0}$ is $\pi / 2$. For the value $T_{1}=10$ the population of levels of the 3-4 system is 0.25 , and the coherence is optimal for the given intensity of the field and dipole moments $\left(\mu_{i}=1\right)$. The duration of the laser pulse corresponding to this value of the parameter $T_{1}$ is about $155 \mathrm{fs}$, which does not satisfy the necessary requirements on pulse duration. According to Figure 3 for smaller values of the parameter $T_{1}$, corresponding to shorter pulses, the coherence of the 3-4 system is significantly reduced with a simultaneous increase of the coherence of the 1-2 system. Optimal values for the coherence of both the 1-2 and 3-4 systems were found for $T_{1} \leq T$ through a search over different intensities of the field.

In Figure 4 the coherence is plotted as a function of the intensity of the field for parameters $T=3$ and $T_{1}=3$. Coherence $\left|\rho_{34}\right|$ of the excited 3-4 system is represented by a bold solid line and coherence $\left|\rho_{12}\right|$ of the suppressed 1-2 system by a bold dashed line; thin lines show populations of the upper levels of both two-level systems. For the intense fields, coherence of both systems possess somewhat chaotic structure. Several values of the intensity, e.g., $I_{0}=$ 
$1.8 \pi$ and $I_{0}=2.55 \pi$, give rather low coherence of the 3-4 system but maximum coherence of the 1-2 system. A desired solution for maximum coherence of the 3-4 system is achieved for the intensity coefficient $I_{0}=2 \pi$. This is the result of redistribution of population within that two-level system: half of population is transferred to the upper level providing maximum coherence. The corresponding coherence of the 1-2 system at this intensity is nearly zero, where most population remains in the lower level.

The goal of control of the coherence of two uncoupled two-level systems is achieved with a pulse shape possessing a broad spectral dip at the suppressed frequency and a suitably chosen intensity of the field. This technique allows one to use pulses of duration $T$ to selectively excite transitions having frequency separations $\Delta \omega<1 / T$. Our results should not be taken to imply that one can spectroscopically determine frequencies to better than the inverse temporal width of the pulse. On the other hand, if the frequencies are known from previous measurements, it is possible to suppress one transition and enhance the other by the method outlined above.

\section{Summary}

The article contributes to the field of molecular dynamics controlled by femtosecond laser pulses. The goal of selective excitation of transitions in stimulated Raman scattering with femtosecond pulse shaping is explored in a framework of a semiclassical model of two two-level systems interacting with external field. The conditions for the optical pulse are provided such that the bandwidth is significantly broader than the frequency difference of the transitions and the designed pulse shape fulfills the selective excitation of these transitions. In a weak-field regime the shaped pulse leads to maximum coherence of one two-level system and zero coherence of another. The same effect is achieved in strong fields using a pulse with the proposed shape and a suitably chosen field amplitude.

\section{ACKNOWLEDGMENTS}

The author is grateful to P. H. Bucksbaum and P. R. Berman for valuable discussions. This work was supported by the National Science Foundation
(No. PHY-9987916 and No. PHY-0244841) through the Center for Frontiers in Optical Coherent and Ultrafast Science (FOCUS) and the U.S. Office of Army Research under Grant No. DAAD19-00-10412. The author acknowledges the Alexander von Humboldt Foundation for assistance in presenting this work at the XI International Congress of Quantum Chemistry in Bonn, Germany, 20-26 July 2003.

\section{References}

1. Wan, C.; Fiebig, T.; Schiemann, O.; Barton, J. K.; Zewail, A. H. Proc Natl Acad Sci 2000, 97, 14052.

2. Ihee, H.; Lobastov, V. A.; Gomez, U. M.; Goodson, B. M.; Srinivasan, R.; Ruan, C.-Y.; Zewail, A. H. Science 2001, 291, 458.

3. Pullen, S. H.; Anderson, N. A.; Walker, L. A.; Sension, R. J. J Chem Phys 1998, 108, 556.

4. Hofmann, A.; de Vivie-Riedle, R. J Chem Phys 2000, 112, 5054 .

5. Niikura, H.; Corkum, P. B.; Villeneuve, D. M. Phys Rev Lett 2003, 90, 203601.

6. Drescher, M.; Hentschel, M.; Kienberger, R.; Uiberacker, M.; Yakovlev, V.; Scrinzi, A.; Westerwalbesloh, T.; Lkeineberg, U.; Heinzmann, U.; Krausz, F. Nature 2002, 419, 803.

7. Brabec, T.; Krausz, F. Rev Mod Phys 2000, 72, 545.

8. Quéré, F.; Itatani, J.; Yudin, G. L.; Corkum, P. B. Phys Rev Lett 2003, 90, 073902.

9. Bucksbaum, P. H. Nature 2003, 421, 593.

10. Weinacht, T. C.; White, J. L.; Bucksbaum, P. H. J Phys Chem A 1999, 103, 10166.

11. Pearson, B. J.; White, J. L.; Weinacht, T. C.; Bucksbaum, P. H. Phys Rev A 2001, 63, 063412.

12. Weinacht, T. C.; Bartels, R.; Backus, S.; Backsbaum, P. H.; Pearson, B.; Geremia, J. M.; Rabitz, H.; Kapteyn, H. C.; Murnane, M. M. Chem Phys Lett 2001, 344, 333.

13. Oron, D.; Dudovich, N.; Yelin, D.; Silberberg, Y. Phys Rev Lett 2002, 88, 063004.

14. Scully, M. O.; Kattawar, G. W.; Lucht, R. P.; Opatrny, T.; Pilloff, H.; Rebane, A.; Socolov, A. V.; Zubairy, M. S. Proc Natl Acad Sci 2002, 99, 10994.

15. Gershgoren, E.; Bartels, R. A.; Fourkas, J. T.; Tobey, R.; Murnane, M. M.; Kapteyn, H. C. Opt Lett 2003, 28, 361.

16. Weiner, A. M.; Leaird, D. E.; Wiederrecht, G. P.; Nelson, K. A. Science 1990, 247, 1317

17. Malinovskaya, S. A.; Bucksbaum, P. H.; Berman, P. R. Phys Rev A 2004, 69, 027401.

18. Oron, D.; Dudovich, N.; Yelin, D.; Silberberg, Y. Phys Rev A 2002, 65, 043408.

19. Dudovich, N.; Oron, D.; Silberberg, Y. Nature 2002, 418, 512.

20. Meshulach, D.; Silberberg, Y. Nature 1998, 396, 239.

21. Scully, M. O.; Zubairy, M. S. Quantum Optics; Cambridge University Press: Cambridge, 1997. 